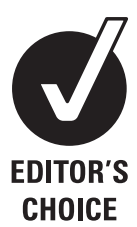

CHOICE

\title{
Respiratory symptoms and inflammatory responses to Difflam throat-spray intervention in half-marathon runners: a randomised controlled trial
}

\author{
A J Cox ${ }^{1,2}$ M Gleeson, ${ }^{2}$ D B Pyne, ${ }^{1}$ P U Saunders, ${ }^{1}$ R Callister, ${ }^{2}$ P A Fricker ${ }^{1}$
}

\begin{abstract}
${ }^{1}$ Australian Institute of Sport, Canberra, Australian Capital Territory, Australia; ${ }^{2}$ School of Biomedical Sciences, University of Newcastle, Newcastle, New South Wales, Australia
\end{abstract}

Correspondence to: Amanda Cox, Department of Physiology, Australian Institute of Sport, PO Box 176, Belconnen, ACT 2616, Australia; amanda.cox@newcastle.edu.au

Accepted 6 May 2008 Published Online First

3 June 2008

\begin{abstract}
Objective: In this study, the effects of Difflam Forte Antiinflammatory Throat Spray on the incidence of upper respiratory symptoms (URS) and inflammatory responses after a half-marathon race were investigated.
\end{abstract}

Design and setting: Double-blind placebo-controlled randomised trial conducted in association with a halfmarathon event.

Participants: 45 well-trained half-marathon runners. Interventions: Difflam ( $n=25)$ or placebo $(n=20)$ throat sprays were self-administered three times daily for 1 week before and 2 weeks after the race.

Main outcome measures: Self-reported respiratory symptoms; plasma prostaglandin $E_{2}$, myeloperoxidase, interleukin (IL) 6, IL8, IL10 and IL1 receptor antagonist (IL1ra) concentrations; and salivary myeloperoxidase and IL6 concentrations.

Results: All subjects completed the intervention without reporting any adverse events. The proportion of athletes reporting URS was not substantially different between Difflam (52\%) and placebo (56\%) groups $(p=0.82)$. However, symptom severity scores were 29\% lower during Difflam treatment (4.7 (7.4) vs 6.6 (9.6)) AU). Postexercise responses in plasma inflammatory markers did not differ substantially between Difflam and placebo groups. Post-race increases in salivary myeloperoxidase ( $63 \%$; trivial to moderate difference; $p=0.13$ ) and salivary IL6 ( 50\%; trivial to moderate difference; $p=0.25$ ) were greater in the Difflam group.

Conclusions: Prophylactic use of the Difflam reduced the severity, but not the frequency, of URS among halfmarathon runners. Post-race increases in systemic inflammatory markers were not altered by Difflam use, but markers of local inflammation (salivary myeloperoxidase and IL6) were augmented in the Difflam compared with the placebo group.

The impairment of athletic performance during periods of upper respiratory symptoms (URS) is a concern for athletes and coaches. The risk for upper respiratory illness in elite athletes is greatest during periods immediately before and after high-level competition, ${ }^{12}$ with some athletes more susceptible than others. The impact of URS on athletic performance has been poorly characterised to date. In a non-exercise setting, there is considerable evidence of impaired pulmonary function during upper respiratory tract infection (URTI). Decreases in respiratory volumes and flow rates ${ }^{3}$ and abnormalities in respiratory muscle function ${ }^{4}$ have been reported during uncomplicated, naturally acquired URTI. There is no evidence to suggest that athletes experiencing URS would be protected from similar changes in pulmonary function. In addition, there is growing evidence confirming disruptions in training schedules among athletes experiencing symptoms of respiratory illness. ${ }^{56}$ Despite these links between illness and performance, there have been few therapeutic intervention trials aimed at preventing or limiting URS in athletes.

Recent studies indicate that not all bouts of URS in athletes have an infectious aetiology. ${ }^{27}$ Accordingly, traditional treatment and management strategies may be ineffective for a proportion of athletes. Examination of the causes of URS in a population of elite athletes revealed that only $\sim 30 \%$ of episodes were associated with an identified respiratory pathogen. ${ }^{7}$ Signs of airway inflammation have been noted in elite athletes in the absence of any underlying pathology, ${ }^{8} 9$ leading to speculation that non-infectious inflammation may contribute to the appearance of URS in some athletes. ${ }^{10}$ This possibility is further supported by evidence demonstrating that a combination antiinflammatory/antibacterial throat spray used after a marathon was able to reduce the post-race incidence and severity of URS in distance runners. ${ }^{2}$

If localised inflammation, in the absence of infection, accounts for the appearance of URS in some athletes, then a topical anti-inflammatory agent may offer some relief. One such product is Difflam Forte Anti-inflammatory Throat Spray, an over-the-counter non-steroidal anti-inflammatory agent (NSAID). The use of a self-administered antiinflammatory throat spray during the weeks before and/or immediately after high-level competition may prove to be an effective prophylactic strategy against URS in athletic populations. There are no reported studies investigating the use of Difflam in alleviating URS in highly trained athletes; however, the product has proven efficacy in relieving pain, soreness and swelling associated with inflammatory conditions of the mouth and throat. ${ }^{11-13}$ There are a number of mechanisms by which the active ingredient in Difflam, benzydamine hydrochloride $(\mathrm{BH})$, exerts its anti-inflammatory actions, including inhibition of the cyclooxygenase $(\mathrm{COX})^{11} 14$ and phospholipase pathways ${ }^{14}$; reduced neutrophil chemotaxis ${ }^{15}$; attenuation of proinflammatory cytokine production ${ }^{16}$; and reduced neutrophil oxidative burst, although it is unclear whether this is a direct consequence of other membrane-stabilising properties. ${ }^{17}$ The measurement of prostaglandins, as end-products of the COX pathway, of proinflammatory (interleukin (IL) 6 and IL8) and anti-inflammatory cytokines 
(IL10 and IL1 receptor antagonist (IL1ra)), and markers of neutrophil activation such as myeloperoxidase (MPO), are likely to be useful in assessing any net effects of Difflam on inflammatory status. ${ }^{16}{ }^{18}$ The aim of this study was to investigate the effectiveness of Difflam in alleviating post-race inflammatory responses and URS in trained runners competing in a half-marathon race.

\section{METHODS}

\section{Subjects and study design}

The study was conducted as a randomised double-blind placebocontrolled trial involving 45 well-trained distance runners participating in the 21.1-km Canberra Half-Marathon (May 2007). Runners were recruited from the local running and triathlon communities and to ensure eligibility for inclusion in the study, interested participants were screened in the 8 weeks before commencement of the trial. Exclusion criteria included a medical history of immune deficiency, liver or kidney disease, severe or aspirin-sensitive asthma, habitual use of antiinflammatory medications and a predicted half-marathon race time exceeding $\sim 100 \mathrm{~min}$ for men and $\sim 110 \mathrm{~min}$ for women. Demographic information for the cohort is shown in table 1. Two runners failed to start the race because of concerns regarding existing injury and were withdrawn from the study at this point. The study was undertaken with approval from the Ethics Committees of the University of Newcastle and the Australian Institute of Sport. The study was designed with consideration of the CONSORT Statement on randomised controlled trials. ${ }^{19}$ All subjects provided written informed consent before participation. Athletes were not required to alter their training, pre-race routine or dietary practices in any way. The experimental design is illustrated in fig 1. Given the short duration of the treatment period ( 3 weeks), interim analyses were not included in the design.

\section{Treatment allocations}

Athletes provided their personal-best half-marathon race time as an indicator of performance ability and were matched on predicted race times. To ensure that the proportion of male and female athletes in each of the treatment groups was similar, female athletes were paired only with other female athletes, and likewise, male athletes were paired only with other male athletes. One member from each pair was then randomly allocated to the Difflam treatment group (DIF), the other to the placebo group (PLA). The active and placebo sprays were manufactured specifically for the trial (iNova Pharmaceuticals, Sydney, Australia) and were identical in appearance with the exception of a coded identification number for each treatment. Simple random allocation of athletes from each pair into the two treatment groups was completed independently of the research team. Treatment identification numbers were held by the manufacturer until sample collection and analysis were completed.

Runners in the DIF group $(n=25)$ were provided with Difflam (iNova Pharmaceuticals) containing $0.3 \%$ benzydamine hydrochloride. Runners from the PLA group $(n=20)$ were provided with a placebo throat spray (iNova Pharmaceuticals) identical in composition to the Difflam except for the omission of the active ingredient (benzydamine hydrochloride). Athletes self-administered the throat spray for 1 week before and 2 weeks after the half-marathon. Athletes were provided with written and verbal instructions to deliver four sprays, three times daily (morning, afternoon and night) directly onto the oropharynx. Difflam Forte and placebo sprays were weighed before and after the intervention period and a total dose for each subject estimated based on the change in weight of the sprays.

\section{Sample collection}

Blood samples were collected from runners $\sim 24$ h pre-race and again immediately post-race. Samples were collected directly into $\mathrm{K}_{3}$ EDTA and lithium heparin collection tubes (Greiner Bioone; Frickenhausen, Germany). Plasma was separated by centrifugation at $3000 \mathrm{rpm}$ and $4^{\circ} \mathrm{C}$ for $8 \mathrm{~min}$ and stored frozen at $-80^{\circ} \mathrm{C}$ until analysis. Whole, mixed, unstimulated saliva $(\sim 1 \mathrm{ml})$ was collected from subjects by passive drool directly into collection tubes. Subjects were directed to avoid bringing saliva, phlegm or sputum forward from the back of the throat. Samples were frozen immediately after collection and were stored at $-80^{\circ} \mathrm{C}$ until analysis.

\section{IIIness records}

Athletes maintained a prospective illness record across the 6 weeks of the study to indicate the presence of any illness symptoms. The illness record was designed at the Australian Institute of Sport and has been used previously in similar study designs. ${ }^{20}$ An episode of URS was recorded if symptoms persisted for two or more consecutive days. In addition to indicating the type of symptoms experienced, athletes also graded the symptom severity based on the impact on training. Symptoms were given a score of 1, if mild in severity (normal training); a score of 2, if moderate in severity (modified training); and a score of 3 , if severe (discontinued training). Global episode severity scores were calculated by totalling daily severity scores for the duration of the episode.

\section{Plasma prostaglandin $E_{2}$ and myeloperoxidase}

The concentration of prostaglandin $\mathrm{E}_{2}\left(\mathrm{PGE}_{2}\right)$ concentrations in $\mathrm{K}_{3}$ EDTA plasma samples was determined using commercially available enzyme immunoassay kits (Amersham Biosciences, Buckinghamshire, UK). Samples were pretreated with $0.4 \mathrm{M}$ indomethacin (Sigma-Aldrich, St Louis, Missouri, USA) immediately after collection to prevent ex vivo $\mathrm{PGE}_{2}$ production. The concentration of myeloperoxidase (MPO) in lithium heparin plasma samples was determined using commercially available enzyme immunoassay kits (HyCult Biotechnology, Uden, The Netherlands). Both $\mathrm{PGE}_{2}$ and $\mathrm{MPO}$ assays were completed according to the manufacturers' instructions. The coefficients of variation (CV) for the low and high controls, respectively, were $\mathrm{PGE}_{2}: 11.6 \%$ and $5.8 \%$, and $\mathrm{MPO}: 9.4 \%$ and $4.4 \%$. All samples

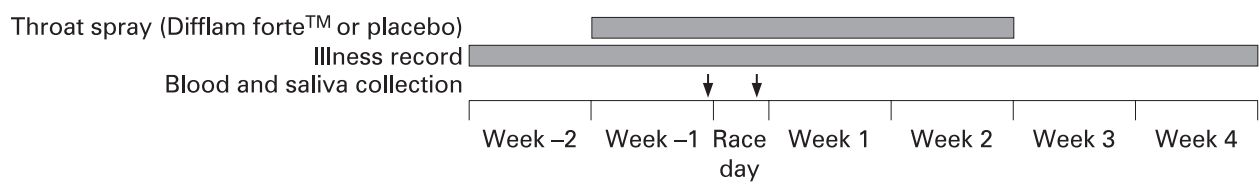

Figure 1 The study design included a 3-week treatment period with either a Difflam or placebo throat spray. Blood and saliva samples were collected $\sim 24 \mathrm{~h}$ pre-race and immediately post-race. A prospective illness record was maintained for the duration of the study. 
Table 1 Demographic and performance characteristics for runners in Difflam and placebo groups, including previous best half-marathon (HM) race time and performance time achieved in the Canberra Half-Marathon (CHM)

\begin{tabular}{lcc}
\hline & $\begin{array}{c}\text { Difflam group } \\
\mathbf{n = 2 5}\end{array}$ & $\begin{array}{l}\text { Placebo group } \\
\mathbf{n}=\mathbf{2 0}\end{array}$ \\
\hline Male:female (n) & $20: 5$ & $16: 4$ \\
Age (years) & $35.1(7.7)$ & $35.2(8.4)$ \\
Height (cm) & $177.6(11.2)$ & $176.7(8.6)$ \\
Mass (kg) & $72.5(12.2)$ & $72.4(12.1)$ \\
Years of training & $8.9(9.1)$ & $8.1(7.1)$ \\
Weekly training load (h week $\left.{ }^{-1}\right)$ & $9.7(5.1)$ & $10.7(4.1)$ \\
Previous best HM time (min) & $88.6(10.8)$ & $87.8(12.3)$ \\
CHM race time (min) & $91.4(10.7)$ & $88.9(10.8)$ \\
\hline
\end{tabular}

Data are presented as mean (SD).

from individual subjects were analysed in a single assay to avoid any inter-assay variation.

\section{Plasma cytokines}

Plasma concentrations of IL6, IL8, IL10 and IL1ra were determined simultaneously using a Bio-Plex Suspension Array System (Bio-Rad Laboratories, Hercules, California, USA) and custom-manufactured Multiplex Cytokine Kits (Bio-Rad Laboratories) according to the manufacturer's instructions. The CV for the low and high controls, respectively, were IL6: 7.2\% and 5.3\%; IL8: $7.7 \%$ and $7.1 \%$; IL10: $6.3 \%$ and $6.9 \%$; and IL1ra: $11.7 \%$ and $3.2 \%$. All samples from individual subjects were analysed in a single assay to avoid any inter-assay variation.

Figure 2 Flowchart detailing progression of participants through the trial, including reasons for exclusion and losses at follow-up.

\section{Salivary MPO and IL6}

The concentration of MPO in saliva was determined using commercially available enzyme immunoassay kits (BioCheck, Foster City, California, USA). The concentration of IL6 in saliva samples was determined using commercially available highsensitivity enzyme immunoassay kits (R\&D Systems, Minneapolis, Minnesota, USA), as described previously. ${ }^{21}$ The coefficients of variation (CV) for the low and high controls, respectively, were MPO: $8.1 \%$ and 5.5\%; and IL6: $3.4 \%$ and $6.1 \%$. All samples from individual subjects were analysed in a single assay to avoid any inter-assay variation.

\section{Statistical analysis}

An analysis combining traditional statistical methods and magnitude-based inferences (effect sizes) and precision of estimation ( $90 \%$ confidence limits) was employed to overcome some of the shortcomings associated with traditional statistical significance testing. ${ }^{22}$ Sample size estimations revealed a sample of 45 distance runners provided adequate power $(>80 \%)$ to detect meaningful differences in key inflammatory markers and a $>50 \%$ difference in the reporting of URS. Descriptive statistics (mean (SD)) were used to summarise the physical and performance characteristics of the athletes. Concentrations of inflammatory markers (blood and saliva) were log-transformed before statistical analysis. Given their large magnitude, postexercise changes in concentrations are reported as a percentage (\%) or "x-fold" changes where appropriate. Race time was included as a covariate in subsequent analyses. $t$ Tests for independent samples and standardised mean changes ${ }^{22}$ were used to compare responses between Difflam and placebo groups

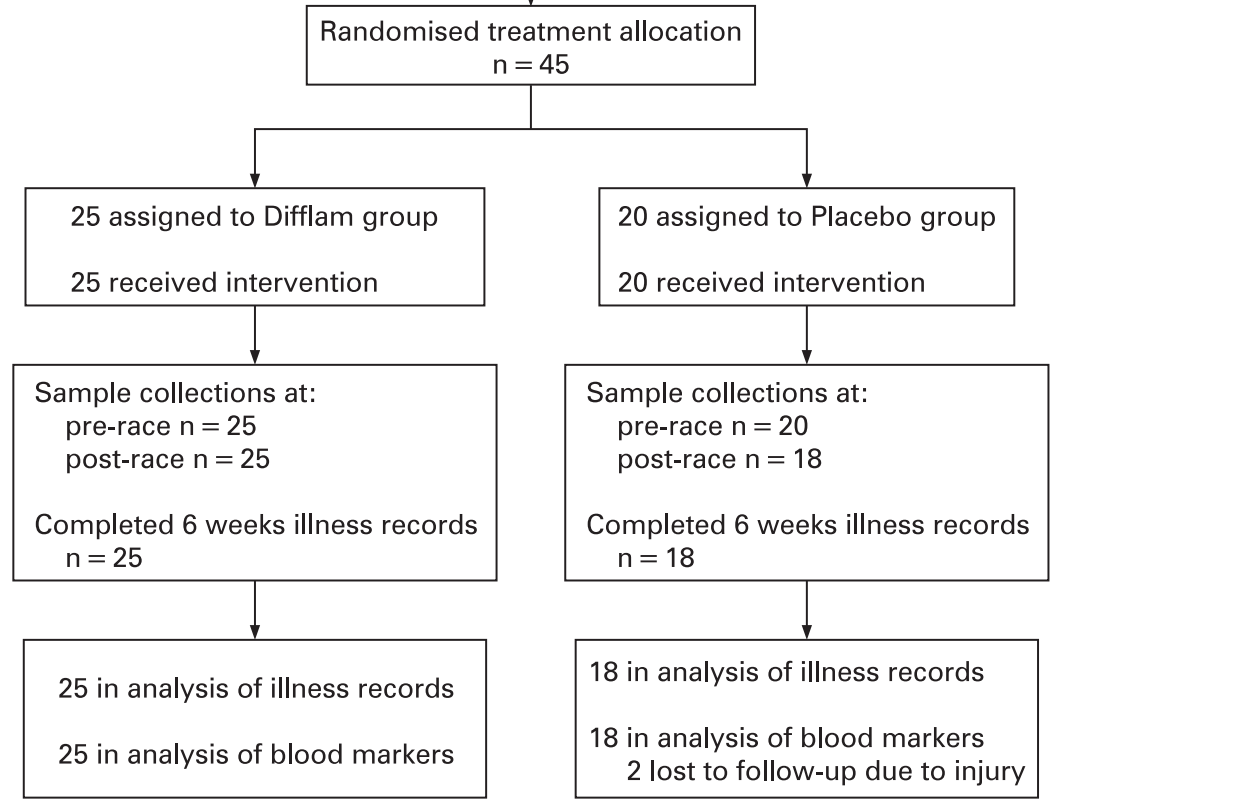

31 excluded
- failed to meet inclusion criteria $(n=4)$

- withdrew due to injury $(n=8)$

- unable to comply with study design $(n=6)$ - declined invitation to participate $(n=13)$ 
Table 2 Episodes of self-reported respiratory symptoms recorded in the Difflam and placebo groups across the entire study and for the 3-week treatment period only.

\begin{tabular}{lcccc}
\hline & Difflam (n= 25) & Placebo (n= 18) & Difference (\%) & $\mathbf{p}$ Value \\
\hline Entire study (6 weeks) & & & & - \\
Number of episodes & 14 & 14 & 8 & 0.82 \\
Proportion of group reporting (\%) & 52 & 56 & 5.6 & 0.84 \\
Mean duration (days) & $3.9(4.9)$ & $4.1(3.7)$ & 9.3 & 0.78 \\
Mean episode severity score (AU) & $4.7(7.3)$ & $5.2(7.3)$ & - & - \\
Treatment period (3 weeks) & 8 & 10 & 56 & 0.23 \\
Number of episodes & 32 & 50 & 23.7 & 0.49 \\
Proportion of group reporting (\%) & $3.8(5.6)$ & $5.0(4.6)$ & 28.9 & 0.45 \\
Mean duration (days) & $4.7(7.4)$ & $6.6(9.6)$ & & \\
Mean episode severity score (AU) & & & \\
\hline
\end{tabular}

Data are presented as mean (SD) where appropriate. The episode severity score is reported in arbitrary units.

(http://sportsci.org/2006/wghcontrial.htm). Criteria for interpreting magnitudes of effects were trivial $0.0-0.2$, small $0.2-0.6$, moderate $0.6-1.2$, large 1.2-2.0 and very large $>2.0$. Effects were deemed to be unclear if the $90 \%$ CI spanned both substantial positive and negative values. Statistical significance for $t$ tests was accepted at $p<0.05$ and below $p=0.001 ; p$ values have been reported as $p<0.001$

\section{RESULTS}

\section{Participant flow}

In keeping with the CONSORT Statement Checklist, participant flow and retention is summarised in fig 2. A number of subjects withdrew their participation between subject pairing and treatment allocation, accounting for the differing numbers of subjects assigned to the DIF and PLA groups.

\section{Race performance and throat spray dose}

The race was conducted on a fine and dry autumn morning. The ambient temperature was $8.2^{\circ} \mathrm{C}$ with $90 \%$ relative humidity at the race start and $14.0^{\circ} \mathrm{C}$ with $68 \%$ relative humidity at the race finish. All 43 participants starting the race completed within $\sim 3.2 \%$ of their predicted race times and completed the intervention without reporting any adverse events. Mean race times were not substantially different $(2.5 \%$ difference; $p=0.46)$ between the DIF (91:24 (10:42) min:s) and PLA groups (88:54 (10:48) min:s). The estimated treatment dose of the throat spray over the 3 weeks of administration was also not significantly different $(3.1 \%$ difference; $p=0.18$ ) between the DIF (33.7 (4.1) g) and PLA groups (30.6 (8.8) g).

\section{Symptoms of upper respiratory illness}

Twenty-three (51\%) of the athletes reported at least one episode of URS over the 6 weeks of the study. Four athletes (9\%) reported URS on more than one occasion. In total, 28 episodes of URS were reported during the study. There was substantial variability in the duration and severity of reported symptoms. The mean episode duration (regardless of intervention group) was 5.3 (4.6 days), with eight of the episodes (29\%) 2 days or less in duration and involving mild, local symptoms only. These presentations are not consistent with the typical presentation of viral URTI.

The proportion of athletes reporting URS across the duration of the study was not appreciably different between DIF and PLA groups $(p=0.89)$ (table 2$)$. Similarly, rates of reporting were not different between groups during the 3 weeks of throat spray use $(p=0.37)$ (table 2). Over the entire 6 weeks of the study,

Table 3 Concentrations of plasma $\mathrm{PGE}_{2}$ and both plasma and salivary (sal) MPO and cytokines in the Difflam and placebo groups pre-race and post-race

\begin{tabular}{|c|c|c|c|c|c|c|c|}
\hline & \multirow[b]{2}{*}{ Group } & \multicolumn{2}{|l|}{ Concentration } & \multicolumn{4}{|c|}{ Outcome statistics } \\
\hline & & Pre & Post & $\%$ Change & $\mathrm{p}$ Value & ES $(90 \% \mathrm{Cl})$ & Descriptor \\
\hline \multirow[t]{2}{*}{$\mathrm{PGE}_{2}\left(\mathrm{pg} \mathrm{ml^{-1 } )}\right.$} & DIF & $230.6(139 \%)$ & $390.8(74 \%)$ & 70 & 0.02 & $0.71(0.47)$ & Small-moderate \\
\hline & PLA & $259.9(149 \%)$ & $293.8(142 \%)$ & 13 & 0.63 & $0.13(0.54)$ & Unclear \\
\hline \multirow[t]{2}{*}{ MPO (ng ml-1) } & DIF & $75.0(61 \%)$ & $318.9(89 \%)$ & 325 & $<0.001$ & $2.54(0.47)$ & Very large \\
\hline & PLA & $71.7(62 \%)$ & $271.9(63 \%)$ & 280 & $<0.001$ & $2.69(0.54)$ & Very large \\
\hline \multirow[t]{2}{*}{ IL6 $\left(p g \mathrm{ml}^{-1}\right)$} & DIF & $2.1(195 \%)$ & $19.3(54 \%)$ & 841 & $<0.001$ & $2.66(0.47)$ & Very large \\
\hline & PLA & $2.0(105 \%)$ & $15.7(73 \%)$ & 696 & $<0.001$ & $3.18(0.53)$ & Very large \\
\hline \multirow[t]{2}{*}{ IL8 $\left(p g \mathrm{ml}^{-1}\right)$} & DIF & $2.9(75 \%)$ & $13.7(39 \%)$ & 366 & $<0.001$ & $3.28(0.47)$ & Very large \\
\hline & PLA & $3.2(66 \%$ & $12.9(61 \%)$ & 306 & $<0.001$ & $2.78(0.5)$ & Very large \\
\hline \multirow[t]{2}{*}{ IL10 $\left(p g \mathrm{ml}^{-1}\right)$} & DIF & $1.8(88 \%)$ & $26.2(99 \%)$ & 1337 & $<0.001$ & $3.98(0.48)$ & Very large \\
\hline & PLA & $2.0(49 \%$ & $21.6(102 \%)$ & 980 & $<0.001$ & $4.04(0.55)$ & Very large \\
\hline \multirow[t]{2}{*}{ IL1ra $\left(\mathrm{pg} \mathrm{ml}^{-1}\right)$} & DIF & $41.3(108 \%)$ & $126.8(55 \%)$ & 207 & $<0.001$ & $1.83(0.47)$ & Large to very large \\
\hline & PLA & $36.9(60 \%)$ & $102.6(49 \%)$ & 178 & $<0.001$ & $2.29(0.54)$ & Large to very large \\
\hline \multirow[t]{2}{*}{ salMPO (ng ml ${ }^{-1}$ ) } & DIF & $304(181 \%)$ & $827(97 \%$ & 172 & $<0.001$ & $1.13(0.47)$ & Moderate to large \\
\hline & PLA & $459(143 \%)$ & $744(126 \%)$ & 72 & 0.09 & $0.56(0.54)$ & Trivial to moderate \\
\hline \multirow[t]{2}{*}{ sallL6 (pg ml-1) } & DIF & $1.2(207 \%)$ & $3.0(150 \%)$ & 156 & 0.003 & $0.90(0.49)$ & Small to large \\
\hline & PLA & $1.3(197 \%)$ & $2.3(116 \%)$ & 62 & 0.09 & $0.56(0.54)$ & Trivial to moderate \\
\hline
\end{tabular}

Cl, confidence interval; ES, effect size.

Concentrations are back-transformed means (CV \%). Outcome statistics relate to the percentage change in concentrations from pre-race to post-race. 

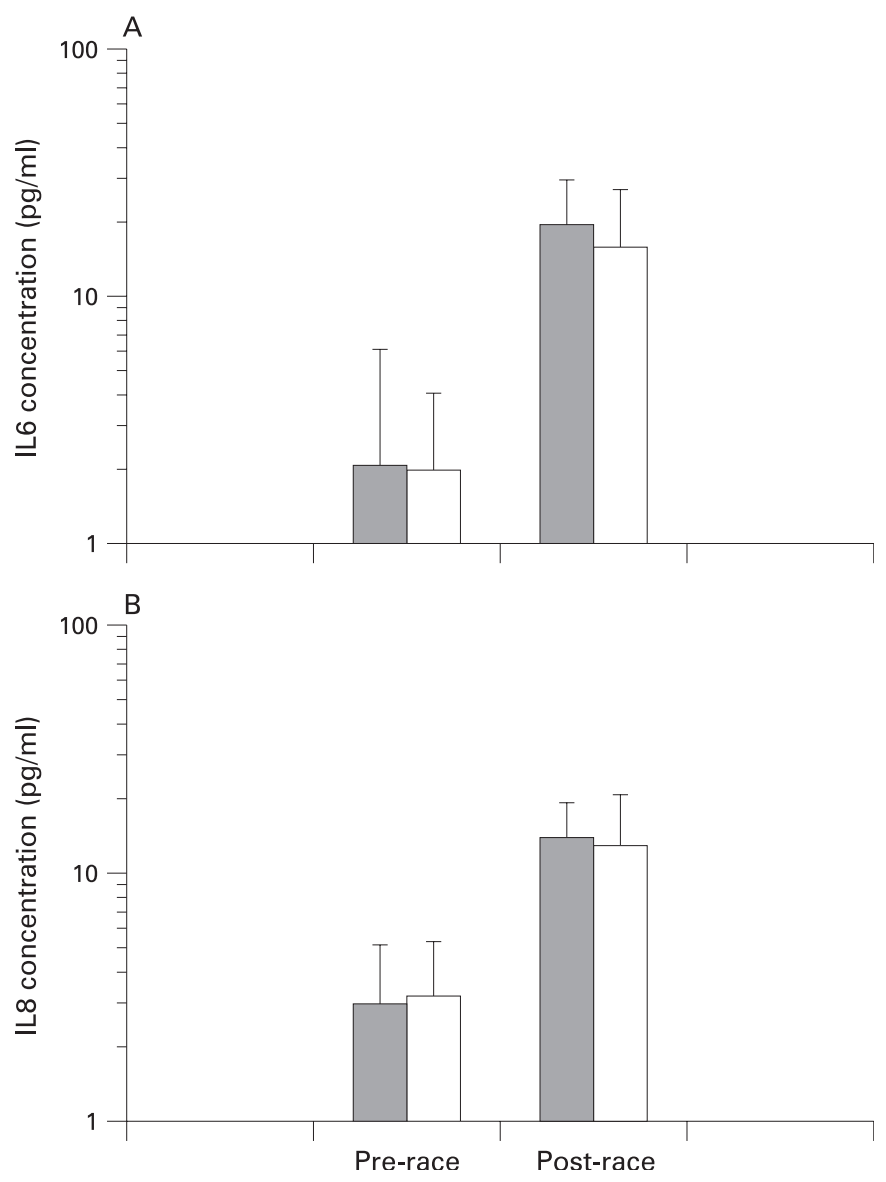

Figure 3 (A) IL6 and (B) IL8 concentrations pre-race and post-race for Difflam ( $\square$ ) and placebo $(\square)$ groups. Vertical bars represent SDs.

episode duration and severity scores did not differ between DIF and PLA groups (table 2). However, during the 3-week treatment period, there was a trend for reduced episode severity among the athletes receiving the Difflam compared with placebo (severity scores 4.7 vs $6.6 \mathrm{AU} ; \sim 29 \%$ difference).

\section{Plasma $\mathrm{PGE}_{2}$ and MPO concentrations}

There was a substantial increase in mean plasma $\mathrm{PGE}_{2}$ concentrations among the DIF group immediately post-exercise $(70 \%$; small to moderate; $\mathrm{p}=0.02)$. In contrast, mean $\mathrm{PGE}_{2}$ concentrations were not substantially elevated post-exercise in the PLA group $(p=0.63)$. There were substantial increases in mean plasma MPO concentrations among both the DIF (4.3fold; very large; $\mathrm{p}<0.001$ ) and PLA (3.8-fold; very large; $p<0.001)$ groups immediately post-exercise. The magnitude of post-exercise increases was not significantly different between the groups $(12 \%, p=0.53)$.

\section{Plasma cytokine concentrations}

There were substantial increases in mean IL6, IL8, IL10 and IL1ra concentrations post-race for both DIF and PLA groups (table 3). The magnitudes of increase in IL6 concentrations postexercise was not substantially different $(7.8 \% ; p=0.81)$ between DIF and PLA groups (9.4-fold vs 8.7-fold, respectively) (fig 3A). Likewise, post-exercise increases in IL8 concentrations were not substantially different $(7.1 \% ; p=0.32)$ between DIF and PLA groups (4.7-fold versus 3.9-fold, respectively) (fig 3B). In contrast, post-exercise increases in IL10 concentrations tended to be greater among the DIF (14.4-fold) compared with
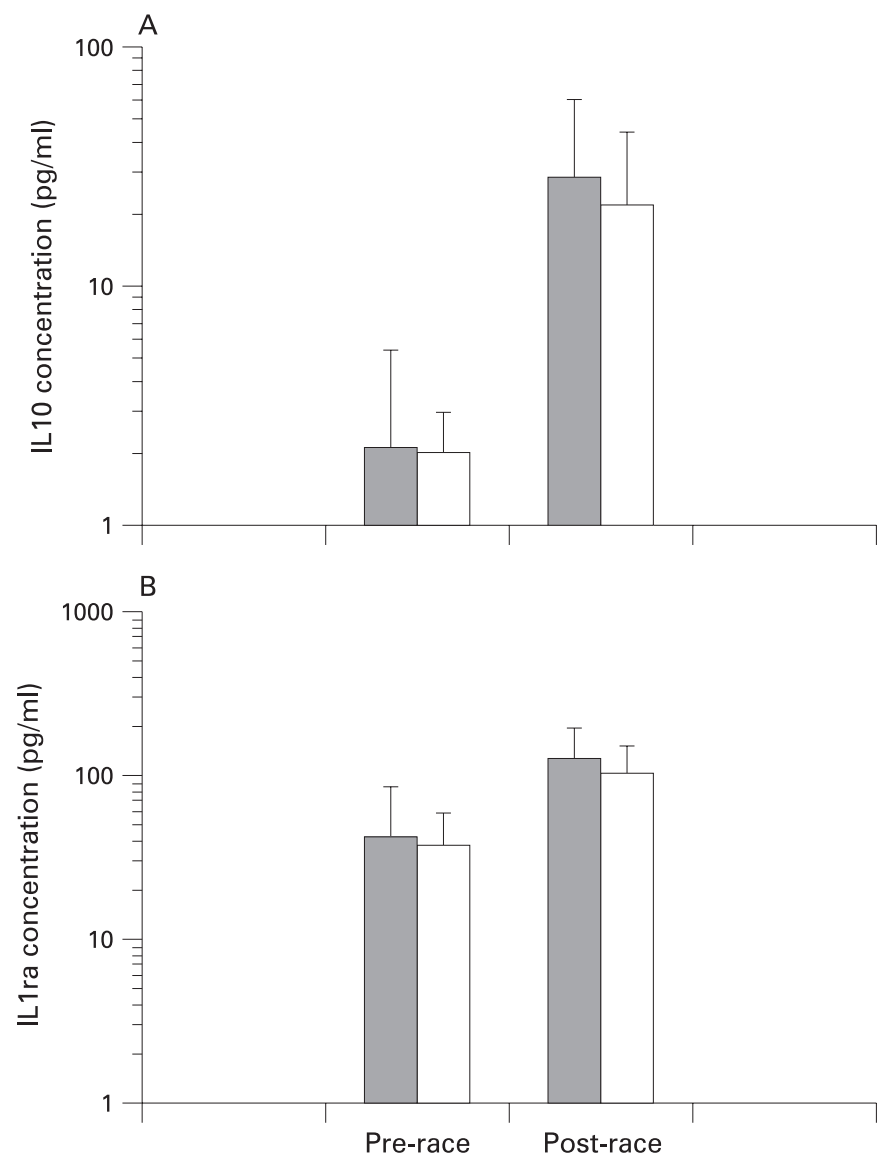

Figure 4 (A) IL10 and (B) IL1ra concentrations pre- and post-race for Difflam ( $\square$ ) and placebo ( $\square$ ) groups. Vertical bars represent SDs.

the PLA (11.7-fold) group (23\%; $p=0.40$; trivial to moderate difference) (fig 4A). The magnitudes of increase in IL1 ra concentrations were not substantially different $(5.9 \%$; $p=0.76$ ) between the DIF and PLA groups (3.1-fold vs 2.9-fold, respectively) (fig 4B).

\section{Salivary MP0 and IL6 concentrations}

There were substantial increases in mean concentrations of salivary MPO (salMPO) and IL6 (salIL6) post-exercise for both DIF and PLA groups (table 3). Post-exercise increases in salMPO concentrations were substantially greater $(63 \% ; p=0.13$, trivial to moderate difference) among the DIF (2.6-fold) compared with the PLA group (1.6-fold) (fig 5A). Similarly, post-exercise increases in sallL6 concentrations were substantially greater $(50 \% ; p=0.25$; trivial to moderate difference) among the DIF (2.5-fold) compared with the PLA group (1.7-fold) (fig 5B).

\section{DISCUSSION}

The prophylactic use of Difflam did not alter the incidence of URS in trained distance runners preparing for and completing a half-marathon race. However, in keeping with the proven efficacy of Difflam in a range of clinical settings, ${ }^{11-13}$ symptom severity scores were reduced with Difflam use. Given there are few published studies examining the effectiveness of treatment or management strategies for URS in athletes, these findings have direct relevance for distance runners, particularly those who experience URS around competition periods. Further, the data offer sports physicians an alternative approach for treating URS in athletic populations. 

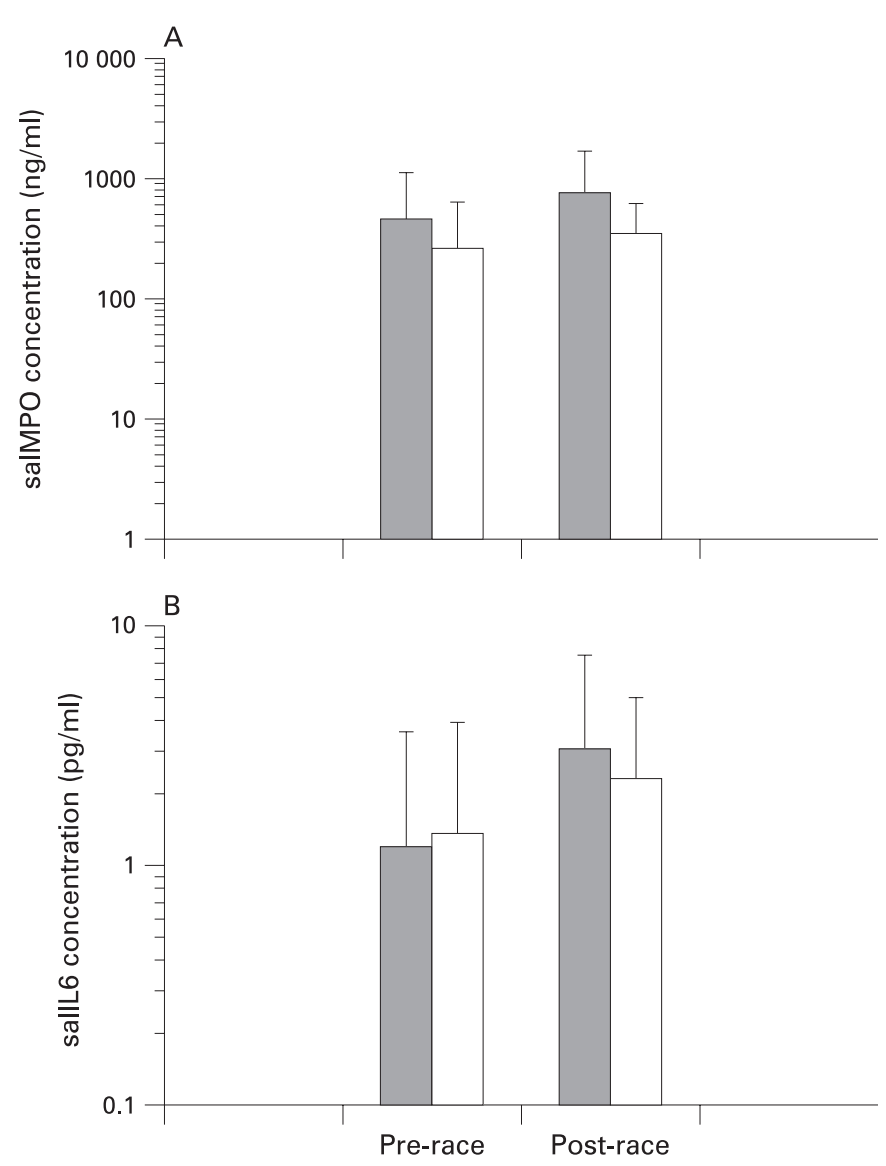

Figure 5 (A) Salivary myeloperoxidase and (B) salivary IL6 concentrations pre-race and post-race for Difflam ( $\square$ ) and placebo ( $\square$ ) groups. Vertical bars represent SDs.

The URS reported in the current study varied in nature. Approximately $30 \%$ of the self-reported episodes were of very short duration (2 days or less) and involved only localised, mild symptoms. It is unlikely that these short episodes were associated with an infectious aetiology. This finding is consistent with other reports in which a considerable proportion of episodes of URS in elite athletes were not associated with an identified infectious aetiology. ${ }^{7}$ It is likely that these symptoms result from ongoing mechanical damage to the mucosal surfaces of the upper airways as a consequence of the increased ventilatory loads during intense exercise and a subsequent inflammatory response. Although the prophylactic administration of Difflam is unable to prevent mechanical damage to the upper airways, relief from the subsequent sequelae of a localised inflammation is plausible. The usefulness of Difflam in alleviating URS in other athletic populations, where mechanical damage to the upper airways is less likely, warrants further investigation.

Despite the variable pattern of reported symptoms, the reduced symptom severity observed in response to Difflam is consistent with the previously demonstrated clinical benefits of $\mathrm{BH}$ treatment. ${ }^{11-13}$ There did not appear to be any residual carryover effects in the 2 weeks after the cessation of Difflam use, which can be explained by the short half-life of $\mathrm{BH}$ after topical administration. ${ }^{17}$ In the current study, a prophylactic approach was favoured over a simple treatment approach where differences in the duration of treatment and the onset of treatment relative to the onset of symptoms were identified as potential confounding variables. However, it is likely that reduced symptom severity would also be observed when throat sprays are used in accordance with traditional prescription guidelines, that is, at the onset of and for the duration of respiratory symptoms. While a treatment rather than a prophylactic approach is more practical for competing athletes, all athletes in the current study completed the prophylactic dosing regimen without any adverse clinical or performance outcomes supporting the safe use of Difflam in athletic populations.

The substantial post-exercise increases in plasma cytokine concentrations were consistent with reported responses to events of similar duration. ${ }^{23}{ }^{24}$ Alterations in plasma MPO in response to exercise have been less well-characterised, ${ }^{25}$ but the large increases after the half-marathon are consistent with known changes in neutrophil degranulation post-exercise. ${ }^{26} 27$ Prostaglandin responses were highly variable between individuals preventing any clear conclusions. Few studies have examined in vivo changes in inflammatory markers after topical $\mathrm{BH}$ treatment in humans, with most focused solely on clinical outcomes. ${ }^{11}{ }^{13}$ Studies that provide evidence of attenuated inflammatory responses after $\mathrm{BH}$ treatment have been completed in animal models $s^{28}$ or in vitro systems. ${ }^{14}{ }^{15}$ In the current study, the apparent lack of effect of Difflam treatment on plasma cytokine and MPO responses may reflect that the effects of $\mathrm{BH}$ are mediated only at a local rather than a systemic level, in line with the topical application of the throat spray.

In contrast to systemic responses, exercise-induced changes in local inflammatory markers in saliva were augmented with Difflam treatment. This observation is counterintuitive, although it is similar to reports of greater post-exercise changes in plasma cytokines and oxidative stress markers after use of NSAID. ${ }^{18}$ 29-31 Altered prostaglandin production after NSAID treatment may disrupt feedback inhibition mechanisms resulting in increased production of downstream inflammatory mediators. $^{32}{ }^{33}$ Given that the mechanisms of action of $\mathrm{BH}$ include reduced prostaglandin synthesis, ${ }^{13}{ }^{14}$ disrupted feedback inhibition may be one explanation for the augmented local responses observed in the current study.

The augmented local inflammatory responses observed postexercise with Difflam treatment raise interesting questions about the mechanisms contributing to the observed reduction in the severity of URS in this cohort of distance runners. It was anticipated that the topical administration of the BH would attenuate any increases in local pro-inflammatory cytokine concentrations, ${ }^{16}$ mediating a clinical benefit via a reduction in localised inflammation. While it remains possible that local inflammatory responses may have been attenuated during acute episodes of symptoms in the Difflam Forte-treated group, this was not measured directly in the current study. It is also interesting to consider the augmented local proinflammatory response in conjunction with documented evidence that proinflammatory cytokines, through interaction with the central nervous system, induce the classic "sickness behaviours" typically seen in response to infection. ${ }^{34}$ Sickness behaviours include malaise, lethargy, loss of appetite and changes in sleep and are recognised as an organised response to help fight infection. ${ }^{35} 36$ The augmented pro-inflammatory cytokine responses may therefore have the potential to contribute to an improved clinical outcome and may simply reflect the classic stress response.

In conclusion, the current investigation of the effects of Difflam on respiratory symptoms and inflammatory responses in half-marathon runners identified the potential for Difflam Forte to reduce the severity, but not the incidence of URS. The 


\section{What is already known on this topic}

- Not all episodes of upper respiratory symptoms in athletic populations appear to have an infective cause.

- Few studies have examined the efficacy of anti-inflammatorybased interventions in alleviating the severity of post-race upper respiratory symptoms.

\section{What this study adds}

Anti-inflammatory-based interventions may be useful in alleviating the severity of post-race upper respiratory symptoms in athletic populations.

- Effective prevention of upper respiratory symptoms in athletic populations must target the cause of the symptoms.

precise mechanisms underpinning the reduction in symptom severity remain unclear, but are likely to be mediated at the local level, consistent with the topical application of the product.

Acknowledgements: The authors gratefully acknowledge the supply of Difflam Forte Anti-inflammatory Throat Spray and placebo throat sprays from iNova Pharmaceuticals (Sydney, Australia). The willing participation of the athletes, the support of Mr Ken Eynon (ACT Cross Country Club, Canberra, Australia) and the technical assistance provided by staff members from the Department of Physiology at the Australian Institute of Sport are also appreciated.

Funding: This project was funded by a research grant from the Australian Institute of Sport (Australian Sports Commission).

Competing interests: None.

\section{REFERENCES}

1. Nieman DC, Dumke Cl, Henson DA, et al. Immune and oxidative changes during and following the western states endurance run. Int J Sports Med 2003;24:541-7.

2. Schwellnus $\mathbf{M}$, Kiessig $\mathbf{M}$, Derman $\mathbf{W}$, et al. Fusafungine reduces symptoms of upper respiratory tract infections (URTI) in runners after a $56 \mathrm{~km}$ race. Med $\mathrm{Scl}$ Sports Exerc 1997:S296.

3. Walter S. Effect of upper respiratory infections on the forced expiratory volumes and flow rates. Indian J Physiol Pharmacol 1989;33:253-4.

4. Mier-Jedrzejowicz A, Brophy C, Green M. Respiratory muscle weakness during upper respiratory tract infections. Am Rev Respir Dis 1988;138:5-7.

5. Weidner TG. Reporting behaviours and activity level of intercollegiate athletes with an URI. Med Sci Sports Exerc 1994;26:22-6.

6. Strauss RH, Lanese RR, Leizman DJ. Illness and absence among wrestlers, swimmers and gymnasts at a large university. Am J Sports Med 1988;16:653-5.

7. Spence L, Brown WJ, Pyne DB, et al. Incidence, etiology, and symptomatology of upper respiratory illness in elite athletes. Med Sci Sports Exerc 2007;39:577-86.

8. Morici G, Bonsignore MR, Zangla D, et al. Airway cell composition at rest and after an all-out test in competitive rowers. Med Sci Sports Exerc 2004;36:1723-9.

9. Bonsignore MR, Morici G, Riccobono L, et al. Airway cells after swimming outdoors or in the sea in nonasthmatic athletes. Med Sci Sports Exerc 2003;35:1146-52.
10. Karjalainen E, Laitinen A, Sue-Chu M, et al. Evidence of airway inflammation and remodeling in ski athletes with and without bronchial hyperresponsiveness to methacholine. Am J Respir Crit Care Med 2000;161:2086-91.

11. Kati I, Tekin M, Silay E, et al. Does benzydamine hydrochloride applied preemptively reduce sore throat due to laryngeal mask airway? Anesth Analg 2004;99:710-2.

12. Young JR. A comparative study of benzydamine hydrochloride ("Difflam" pump spray) and placebo as analgesics following tonsillectomy. Int J Tissue React 1987:9:131-3.

13. Epstein JB, Silverman S, Paggiarino DA, et al. Benzydamine $\mathrm{HCl}$ for prophylaxis of radiation-induced oral mucositis. Cancer 2001;92:875-85.

14. Modeer T, Yucel-Lindberg T. Benzydamine reduces prostaglandin production in human gingival fibroblasts challenged with interleukin-1 $\beta$ or tumor necrosis factor- $\alpha$. Acta Odontol Scand 1999;57:40-5.

15. Riboldi E, Frascaroli G, Transidico P, et al. Benzydamine inhibits monocyte migration and MAPK activation induced by chemotactic agonists. Br J Pharmacol 2003:140:377-83

16. Sironi M, Pozzi P, Polentarutti N, et al. Inhibition of inflammatory cytokine production and protection against endotoxin toxicity by benzydamine. Cytokine 1996:8:710-6.

17. Quane PA, Graham GG, Ziegler JB. Pharmacology of benzydamine. Inflammopharmacology 1998;6:95-107.

18. Pizza FX, Cavender D, Stockard A, et al. Anti-inflammatory doses of ibuprofen: effect on neutrophils and exercise-induced muscle injury. Int J Sports Med 1999;20:98-102.

19. Altman DG, Schultz KF, Moher D, et al. The revised CONSORT statement for reporting randomized trials: explanation and elaboration. Ann Intern Med 2001:134:663-94.

20. Cox AJ, Gleeson M, Pyne DB, et al. Valtrex ${ }^{\mathrm{TM}}$ therapy for Epstein-Barr virus reactivation and upper respiratory symptoms in elite runners. Med Sci Sports Exerc 2004;36:1104-10.

21. Minetto M, Rainoldi A, Gazzoni M, et al. Differential responses of serum and salivary interleukin-6 to acute strenuous exercise. Eur J Appl Physiol 2005;93:679-86.

22. Batterham AM, Hopkins WG. Making meaningful inferences about magnitudes. Int J Sports Physiol Perform 2006;1:50-7.

23. Nieman DC, Henson DA, Davis MJ, et al. Blood leukocyte mRNA expression for IL10, IL-1ra, and IL-8, but not IL-6, increases post-exercise. J Interferon Cytokine Res 2006;26:668-74.

24. Peake JM, Suzuki K, Hordern M, et al. Plasma cytokine changes in relation to exercise intensity and muscle damage. Eur J App/ Physiol 2005;95:514-21.

25. Bury TB, Pirnay F. Effect of prolonged exercise on neutrophil myeloperoxidase secretion. Int J Sports Med 1995:16:410-2.

26. Bishop NC, Walsh NP, Haines DL, et al. Pre-exercise carbohydrate status and immune responses to prolonged cycling: I. Effect on neutrophil degranulation. Int J Sport Nutr Exerc Metab 2001;11:490-502.

27. Lancaster GI, Jentjens RL, Moseley L, et al. Effect of pre-exercise carbohydrate ingestion on plasma cytokine, stress hormone, and neutrophil degranulation responses to continuous, high-intensity exercise. Int J Sport Nutr Exerc Metab 2003;13:436-53.

28. Guglielmotti A, Aquilini L, Rosignoli MT, et al. Benzydamine protection in a mouse model of endotoxemia. Inflamm Res 1997:46:332-5.

29. Nieman DC, Dumke CL, Henson DA, et al. Muscle damage is linked to cytokine changes following a 160-km race. Brain Behav Immun 2005:19:398-403.

30. Nieman DC, Henson DA, Dumke CL, et al. Ibuprofen use, endotoxemia, inflammation, and plasma cytokines during ultramarathon competition. Brain Behav Immun 2006;20:578-84

31. McAnulty SR, Owens JT, McAnulty LS, et al. Ibuprofen use during extreme exercise: effects on oxidative stress and PGE 2 . Med Sci Sports Exerc 2007;39:1075-9.

32. Rhind SG, Gannon GA, Shephard RJ, et al. Indomethacin modulates circulating cytokine responses to strenuous exercise in humans. Cytokine 2002;19:153-8.

33. Hartel C, vonPuttkamer J, Gallner F, et al. Dose-dependent immunomodulatory effects of acetylsalicylic acid and indomethacin in human whole blood: potential role of cyclooxygenaze-2 inhibition. Scand J Immunol 2004;60:412-20.

34. Smith LL. Cytokine hypothesis of overtraining: a physiological adaptation to excessive stress. Med Sci Sports Exerc 2000;32:317-31.

35. Kelley KW, Bluthe R, Dantzer R, et al. Cytokine-induced sickness behavior. Brain Behav Immun 2003;17:S112-8

36. Dantzer R. Cytokine-induced sickness behaviour: a neuroimmune response to activation of innate immunity. Eur J Pharmacol 2004;500:399-411. 